\title{
Dixon Sequence with Superimposed Model-Based Bone Compartment Provides Highly Accurate PET/MR Attenuation Correction of the Brain
}

\author{
Thomas Koesters ${ }^{1,2}$, Kent P. Friedman ${ }^{1,2}$, Matthias Fenchel ${ }^{3}$, Yiqiang Zhan ${ }^{4}$, Gerardo Hermosillo ${ }^{4}$, James Babb ${ }^{1,2}$, \\ Ileana O. Jelescu ${ }^{1,2}$, David Faul ${ }^{4}$, Fernando E. Boada ${ }^{1,2}$, and Timothy M. Shepherd ${ }^{1,2}$ \\ ${ }^{I}$ Bernard and Irene Schwartz Center for Biomedical Imaging, Department of Radiology, New York University School of Medicine, \\ New York, New York; ${ }^{2}$ Center for Advanced Imaging Innovation and Research (CAI2R), New York, New York; ${ }^{3}$ Siemens Healthcare \\ $\mathrm{GmbH}$, Erlangen, Germany; and ${ }^{4}$ Siemens Medical Solutions, USA, Malvern, Pennsylvania
}

Simultaneous PET/MR of the brain is a promising technology for characterizing patients with suspected cognitive impairment or epilepsy. Unlike CT, however, MR signal intensities do not correlate directly with PET photon attenuation correction (AC), and inaccurate radiotracer SUV estimation can limit future PET/MR clinical applications. We tested a novel AC method that supplements standard Dixon-based tissue segmentation with a superimposed modelbased bone compartment. Methods: We directly compared SUV estimation between MR-based AC and reference CT AC in 16 patients undergoing same-day PET/CT and PET/MR with a single ${ }^{18} \mathrm{~F}-\mathrm{FDG}$ dose for suspected neurodegeneration. Three Dixonbased MR AC methods were compared with CT: standard Dixon 4-compartment segmentation alone, Dixon with a superimposed model-based bone compartment, and Dixon with a superimposed bone compartment and linear AC optimized specifically for brain tissue. The brain was segmented using a 3-dimensional T1-weighted volumetric MR sequence, and SUV estimations were compared with CT AC for whole-image, whole-brain, and 91 FreeSurferbased regions of interest. Results: Modifying the linear AC value specifically for brain and superimposing a model-based bone compartment reduced the whole-brain SUV estimation bias of Dixon-based PET/MR AC by $95 \%$ compared with reference CT AC $(P<0.05)$, resulting in a residual $-0.3 \%$ whole-brain SUV $_{\text {mean }}$ bias. Further, brain regional analysis demonstrated only 3 frontal lobe regions with an SUV estimation bias of $5 \%$ or greater $(P<$ $0.05)$. These biases appeared to correlate with high individual variability in frontal bone thickness and pneumatization. Conclusion: Bone compartment and linear AC modifications result in a highly accurate MR AC method in subjects with suspected neurodegeneration. This prototype MR AC solution appears equivalent to other recently proposed solutions and does not require additional MR sequences and scanning time. These data also suggest that exclusively model-based MR AC approaches may be adversely affected by common individual variations in skull anatomy.

\footnotetext{
Received Sep. 16, 2015; revision accepted Jan. 5, 2016.

For correspondence or reprints contact: Thomas Koesters, Bernard and Irene Schwartz Center for Biomedical Imaging, New York University School of Medicine, 660 First Ave., New York, NY 10016.

E-mail: thomas.koesters@nyumc.org

Published online Feb. 2, 2016.

COPYRIGHT (C 2016 by the Society of Nuclear Medicine and Molecular Imaging, Inc.
}

Key Words: PET/MR hybrid imaging; MR-based attenuation correction; model-based attenuation correction; attenuation correction of bone

J Nucl Med 2016; 57:918-924

DOI: 10.2967/jnumed.115.166967

I ntegrated PET/MR is a new imaging technology that has many practical benefits for patients, referring physicians, and radiologists and has the potential to affect future clinical and research studies (1). Unfortunately, unlike CT (or earlier rotating transmission sources), MR signal does not provide a direct, linear relationship to electron density that can be used to calculate an attenuation coefficient map ( $\mu$-map) for $511-\mathrm{keV}$ photons to correct for attenuation and scatter in PET $(2,3)$. Currently, attenuation correction (AC) maps in clinical PET/MR studies of the head are derived using the Dixon sequence, which provides up to 4 tissue classes, that is, air, fat, lung, and soft tissue $(4,5)$. However, the Dixon method does not include a bone compartment, leading to an underestimation of SUVs in the brain compared with the reference CT-based AC in $\mathrm{PET} / \mathrm{CT}$. In the brain, inaccurate SUV estimation for various radiotracers from integrated PET/MR may limit its research potential and reduce clinical sensitivity for subtle findings.

Proposed technical improvements in AC for integrated PET/MR systems derive attenuation information either from the PET data or from the MR data. The emission image and the $\mu$-map can be reconstructed simultaneously (6), potentially using prior MR data providing anatomic information. However, a unique solution exists only in cases of time-of-flight PET data. MR-based AC solutions can be divided into segmentation or atlas-based methods. Segmentation approaches assign linear attenuation coefficients (LACs) for different tissue classes after segmentation of a Dixon (4) or ultrashort-echo-time image (7-9). AC approaches based on only Dixon do not account for the bone compartment, that is, the skull. Methods using ultrashort echo time detect bone but may not clearly distinguish bone from airspaces in the skull and sinuses (10). Alternatively, atlas-based methods use anatomic models to deform or supplement a $\mu$-map derived from the MR images of an individual subject. Most such approaches rely on the construction of hypothetical CT data, that is, pseudo-CT images are predicted from MR images (11) and then MR intensities are linked to CT 
Hounsfield units (12) or bone information is transferred from the CT to the MR image after comparing the MR image with an existing database (13).

We report the benefits of a PET/MR AC method that supplements a conventional MR Dixon sequence-derived tissue segmentation with a superimposed model-based bone compartment. This prototype was previously evaluated for whole-body PET/MR scans excluding the brain (14). To evaluate this method, we compared SUV estimation from CT, Dixon, and this model-based approach using both whole-brain and regional analyses in 16 elderly subjects being evaluated for cognitive impairment who underwent serial PET/CT and PET/MR on the same day after a single ${ }^{18} \mathrm{~F}-\mathrm{FDG}$ dose. Our data demonstrate that this new method significantly reduced the wholeand regional-brain SUV estimation bias from Dixon-based MRI.

\section{MATERIALS AND METHODS}

\section{Patient Population}

The local institutional review board approved this study, and informed consent was obtained from all subjects. Sixteen patients (mean age \pm SD, $72.1 \pm 7.5$ y old; range, 58-85 y; 6 female) undergoing clinical head ${ }^{18} \mathrm{~F}-\mathrm{FDG}$ PET/CT for suspected cognitive impairment were recruited to undergo a same-day, repeated-measures comparison head PET/MR examination without additional ${ }^{18} \mathrm{~F}-\mathrm{FDG}$ radiotracer administration. An Alzheimer disease ${ }^{18} \mathrm{~F}-\mathrm{FDG}$ hypometabolism pattern was diagnosed for 11 subjects (69\%). After completion of the study, a board-certified neuroradiologist reviewed the radiology reports, CT images, and MR images to extract subject-specific features that could affect the accuracy of different AC methods. This included the extent of petrous apex, sphenoid, and frontal sinus pneumatization, white matter fluid-attenuated inversion recovery hyperintensities (15), the amount of dental amalgam (ordinal scale), and 3 regions of interest (ROIs for mean CT Hounsfield units in the clivus, basal ganglia, and calvarium).

\section{Imaging Protocol}

The subjects fasted for $4 \mathrm{~h}$ and then were given a single intravenous injection of ${ }^{18} \mathrm{~F}-\mathrm{FDG}(5.18 \mathrm{MBq} / \mathrm{kg}$; mean dose, $366.3 \pm 11.1 \mathrm{MBq})$ after confirmation of a serum glucose level below $200 \mathrm{mg} / \mathrm{dL}$. The patients rested in a quiet room before undergoing a standard clinical PET/CT scan (Biograph mCT; Siemens Healthcare GmbH). From this $\mathrm{PET} / \mathrm{CT}$ acquisition, only the CT images were used. The patients were then transported to a nearby facility for an integrated 3-T PET/MR study (Biograph mMR, software Syngo MR B18P; Siemens Healthcare $\mathrm{GmbH})$. The time from the initial ${ }^{18} \mathrm{~F}-\mathrm{FDG}$ dose administration to imaging was $56.3 \pm 8.7 \mathrm{~min}$ and $156.4 \pm 37.4 \mathrm{~min}$ for PET/CT and PET/MR, respectively. Integrated PET/MR allowed simultaneous acquisition of multiple MR sequences during the PET list-mode acquisition. For anatomic coregistration, a sagittal 3-dimensional magnetizationprepared rapid gradient echo sequence was performed (MPRAGE; repetition, inversion, and echo times $=2,300,900$, and $2.77 \mathrm{~ms}$, respectively; $1.2 \times 1.2 \times 1.3 \mathrm{~mm}$ resolution). Additional multiplanar MR sequences were obtained per the standard clinical protocol.

\section{AC Map Generation}

Dixon $\mu$-Map. The Dixon $\mu$-map reflects the standard 4-compartment $\mu$-map (including air, lung, fat, and soft tissue with LAC values of 0 , $0.0224,0.0854$, and $0.1 \mathrm{~cm}^{-1}$, respectively) from the manufacturer.

CT $\mu$-Map. The CT images acquired with the Biograph mCT were registered to the Dixon $\mu$-map so that all images could be reconstructed from the PET emission data acquired with the Biograph mMR. Rigid registration of the CT to the MR Dixon image was done with self-written registration software using mutual information as a similarity measure. Registration was confirmed by visual inspection. The CT $\mu$-map was then cropped using the MR-based Dixon $\mu$-map to remove from the CT image the patient bed and any objects that were not present during the PET/MR scan; subsequently, the $\mathrm{CT} \mu$-map was transformed from Hounsfield units to LACs at $511 \mathrm{keV}$ (16). Voxels not covered by the CT scan were filled with voxels in the Dixon $\mu$-map to account for potential differences and to avoid influences other than those from differences in the $\mu$-maps.

Bone $\mu$-Map A. The bone attenuation map was computed on the basis of a regular 4-compartment segmentation from a Dixon sequence. Bone information was added to these $\mu$-maps with a model-based bone prototype segmentation algorithm (Siemens Healthcare $\mathrm{GmbH}$ ) using continuous LACs for bone. The segmentation algorithm consisted of off-line (training) and online (runtime) stages. The off-line stage aimed to construct a prealigned MR model image and skull mask pair. The MR model image was carefully aligned and cropped to include only the skull-relevant anatomies. The skull bone masks contain bone densities as LACs in $\mathrm{cm}^{-1}$ at the PET energy level of $511 \mathrm{keV}$. In addition, a set of anatomic landmarks was defined around the skull, and their detectors were trained during the offline stage. Mathematically, the detector of the $i$ th landmark was defined as $C_{i}\left(F_{i}(p)\right)$, where $F_{i}$ and $C_{i}$ denote the image appearance features calculated around voxel $\mathrm{p}$ and a learned Adaboost classifier, respectively. The output of the detector indicates the likelihood that voxel $\mathrm{p}$ belongs to the landmark.

At run time, the MR image of the model was registered with the subject MR image. The registration algorithm consisted of landmarkbased similarity registration and intensity-based deformable registration. In the landmark-based similarity registration, the pretrained detectors are used to detect a set of landmarks surrounding the skull. Specifically, the $i$ th landmark location $p_{i}$ is the voxel with the maximum detector response, defined as Equation 1.

$$
p_{i}=\max _{p \in I} C_{i}\left(F_{i}(p)\right) .
$$

More details can be found in a previous publication (17). These landmarks are used to crop the skull area from the subject MR image in a way similar to that for the model MR image. Afterward, the similarity transformation between the subject and the model is derived from the locations of these landmarks using a least-square solver. After the similarity registration, a more sophisticated deformable registration was performed to bring the model to the subject space.

The algorithm proposed by Hermosillo et al. (18) was used for deformable registration. To achieve diffeomorphic transformation from model to subject, we decompose the overall deformation from model to subject, $\phi_{\mathrm{mdl} \rightarrow \mathrm{sub}}$, into a set of small deformations, that is, $\phi_{m d l \rightarrow s u b}=\phi_{0}{ }^{\circ} \phi_{1}{ }^{\circ \cdots \circ} \phi_{K}$. Each small deformation $\phi_{k}$ is iteratively calculated by Equation 2 .

$$
\phi_{k}=\mathrm{I}+\varepsilon \frac{\partial}{\partial \phi} S\left(\mathrm{MR}_{\mathrm{sub}}, \mathrm{MR}_{\mathrm{mdl}}{ }^{\circ} \phi\right) .
$$

Here, $\phi=\phi_{0}{ }^{\circ} \phi_{1}{ }^{\circ \cdots \circ} \phi_{k-1}$ is the deformation derived by previous iteration. I denotes the identity mapping. S(.) defines the local crosscorrelation between the warped model $\mathrm{MR}, \mathrm{MR}_{\mathrm{mdl}}$, and the subject $\mathrm{MR}, \mathrm{MR}_{\text {sub }}$ (18).

Different Dixon sequence information is used at different stages of the registration framework. Since the first registration stage is based on anatomic landmarks, we select to use fat and out-of-phase sequences, in which the landmarks exhibit more distinctive appearance characteristics. In the second deformable registration stage, we use information from an in-phase Dixon sequence because the cross-correlation calculated from this sequence is more consistent across the population.

The prealigned skull mask is brought to the subject space following the deformation $\phi_{m d l \rightarrow s u b}$. The bone density information is added to 


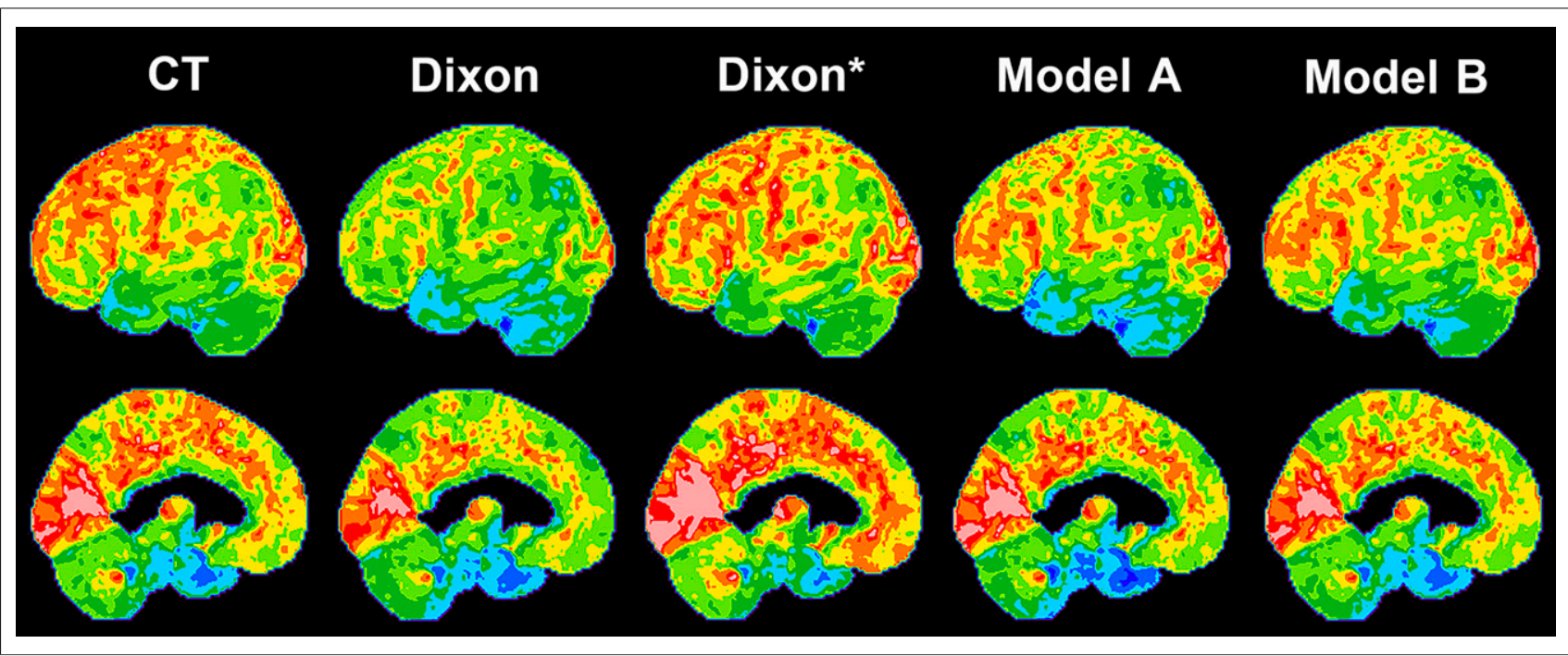

FIGURE 1. Comparison of ${ }^{18} \mathrm{~F}-\mathrm{FDG}$ PET surface maps for left hemisphere in subject with clinical and imaging features consistent with mild Alzheimer dementia (top and bottom rows = lateral and medial surfaces, respectively). ${ }^{18} \mathrm{~F}-\mathrm{FDG}$ surface map using CT AC demonstrates ${ }^{18} \mathrm{~F}-$ FDG hypometabolism in lateral temporal-parietal regions, posterior cingulate, and precuneus (first column). Using same SUV color scale, Dixonbased AC blunts conspicuity of these changes, but overall pattern can be observed once ${ }^{18} \mathrm{~F}-\mathrm{FDG}$ surface is rescaled by expert user (Dixon*). Model-based $\mu$-maps demonstrate ${ }^{18} \mathrm{~F}$-FDG surface maps that are indistinguishable from CT-based attenuation data using same SUV color scale except for subtle differences in the frontal poles. Overall, all $3 \mathrm{MR} \mu$-maps can be used to make appropriate clinical diagnosis.

the original Dixon-based $\mu$-map at all voxels of densities higher than soft tissue after the segmentation process. The average running time of the algorithm was 2-3 min per case on a workstation with a Xeon 2.13-GHz central processing unit (Intel), 2 processors, and $16 \mathrm{~GB}$ of random-access memory (14).

Bone $\mu$-Map B. For bone $\mu$-map B, the LAC for soft tissue was adapted. The original value $\left(0.1 \mathrm{~cm}^{-1}\right)$ was optimal for whole-body 4-compartment $\mu$-maps if the density of soft tissue is averaged throughout the body. We observed brain LACs that were $2 \%$ lower, averaging $0.098 \mathrm{~cm}^{-1}$. Bone $\mu$-map B is identical to bone $\mu$-map A except for this lowered attenuation coefficient for soft tissue.

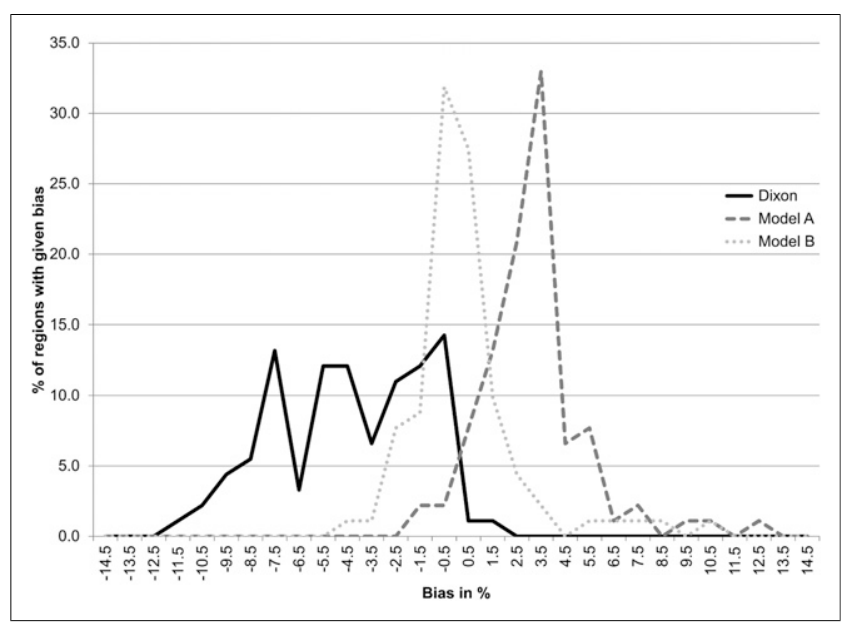

FIGURE 2. Line plot of percentage FreeSurfer regions with given $S U V_{\text {mean }}$ bias for 3 MR AC methods compared with reference CT AC (16 elderly subjects evaluated for dementia, 91 FreeSurfer regions per brain). Anatomic model-based MR AC methods demonstrate narrow line shapes closer to origin, indicating both improved precision and accuracy of SUV estimation.

\section{PET Reconstruction}

From the mMR PET list-mode data, only the first $10 \mathrm{~min}$ for each patient were used. This reduced the chance of artifacts due to patient motion. All PET reconstructions (ordinary Poisson orderedsubsets expectation maximization, 3 iterations and 21 subsets) were performed offline using JSRecon and e7tools provided by Siemens, using a $344 \times 344 \times 127$ matrix with a pixel size of $2.09 \mathrm{~mm}^{2}$ and slice thickness of $2.03 \mathrm{~mm}$. Next to the different human $\mu$-maps, the corresponding hardware $\mu$-maps were used to correct for attenuation and scatter due to the head coil and patient table. Postreconstruction smoothing with a gaussian filter and kernel width of $2 \mathrm{~mm}$ in full width at half maximum was applied.

\section{Data Segmentation and Analysis}

For each patient, 91 ROIs were automatically segmented on the MPRAGE using FreeSurfer, version $5.3(19,20)$. The 45 brain regions for each hemisphere included cerebellar white matter and cortex, thalamus, caudate nucleus, putamen, pallidum, nucleus accumbens, hippocampus, amygdala, and numerous cortical regions (FreeSurfer atlas regions $\mathrm{X} 001-\mathrm{X} 003$ and $\mathrm{X} 005-\mathrm{X} 035, \mathrm{X}=1,2$ for left and right, respectively). The 91st FreeSurfer ROI was the unpaired brain stem. This facilitated analysis to determine which brain regions experienced the largest bias from MR AC errors. The PET reconstructions using the Dixon $\mu$-maps were registered to the MPRAGE using the Oxford Centre for Functional MRI of the Brain Software Library (FLIRT, version 5.5) $(21,22)$ to avoid any misalignment due to patient motion. The calculated transformation matrix was also applied to the PET reconstructions, where the $\mathrm{CT}$, model $\mathrm{A}$, and model $\mathrm{B} \mu$-maps were used. The 91 ROIs were transferred to the PET images, and $\mathrm{SUV}_{\text {mean }}$ was calculated for each region. The percentage deviation in each region for each PET reconstruction with respect to the reference PET reconstruction using the $\mathrm{CT} \mu$-map was calculated.

\section{Statistical Analysis}

Paired-sample Wilcoxon signed-rank tests were used to compare the SUVs derived from the 3 MR methods (Dixon, method A, and method B) 
TABLE 1

Summary of SUV Bias for 3 MR-Based AC Methods Compared with CT AC in 16 Subjects Evaluated for Neurodegeneration

\begin{tabular}{|c|c|c|c|}
\hline SUV bias & Dixon & Model A & Model B \\
\hline Whole-brain bias & $-6.4 \%$ * & $2.4 \%^{*}$ & $-0.3 \%$ \\
\hline Whole-image bias & $-5.9 \% *$ & $2.7 \%^{*}$ & $0.5 \%$ \\
\hline FreeSurfer ROls ${ }^{\dagger}$ & 38 & 13 & 5 \\
\hline Lowest ROI bias & $-11.99 \%$ & $-1.54 \%$ & $-4.29 \%$ \\
\hline Highest ROI bias & $+1.49 \%$ & $+12.03 \%$ & $+10.48 \%$ \\
\hline $\begin{array}{l}\text { Top } 3 \text { regions of absolute } \\
\text { mean bias }\end{array}$ & $\begin{array}{l}\text { Lateral occipital, } \\
\text { inferior parietal, } \\
\text { cerebellar cortex }\end{array}$ & $\begin{array}{l}\text { Pars triangularis, } \\
\text { frontal pole, rostral } \\
\text { middle frontal cortex }\end{array}$ & $\begin{array}{l}\text { Pars triangularis, } \\
\text { frontal pole, rostral } \\
\text { middle frontal cortex }\end{array}$ \\
\hline Asymmetries ${ }^{\ddagger}$ & 6 & 4 & 6 \\
\hline Top 3 regions of asymmetry & $\begin{array}{l}\text { Lateral occipital, } \\
\text { middle temporal, } \\
\text { postcentral cortex }\end{array}$ & $\begin{array}{l}\text { Superior temporal } \\
\text { sulcus, posterior } \\
\text { middle frontal, gyrus } \\
\text { pars triangularis }\end{array}$ & $\begin{array}{l}\text { Superior temporal } \\
\text { sulcus, posterior } \\
\text { middle frontal, } \\
\text { gyrus pars triangularis }\end{array}$ \\
\hline
\end{tabular}

*Significant difference compared with CT-based SUV estimation $(P<0.001)$.

${ }^{\dagger}$ FreeSurfer regions with statistically significant mean bias differences $(P \leq 0.05)$ of at least $5 \%$ (91 FreeSurfer regions studied).

${ }^{\ddagger}$ FreeSurfer regions with statistically significant left-right mean bias differences $(P \leq 0.05)$ of at least $5 \%$ (45 FreeSurfer regions compared between right and left).

with the SUV from CT for the same brain region and patient. In addition, they were also used to compare differences in mean bias between right and left cerebral hemispheres for the 3 MR-based AC methods (relative to reference $\mathrm{CT} \mu$-maps). A secondary analysis was performed to identify subject-specific factors that may correlate with differences in CT and MR $\mathrm{AC}$ methods. Spearman rank correlations characterized the association between these subject-level cofactors and the within-subject difference between the MR and CT SUVs, represented as (MR SUV - CT SUV)/ (CT SUV). All statistical tests were conducted at the 2-sided 5\% significance level using SAS, version 9.3 (SAS Institute).

\section{RESULTS}

\section{MR AC SUV Bias Estimation}

Figure 1 demonstrates typical ${ }^{18} \mathrm{~F}-\mathrm{FDG}$ surface maps from a selected subject in this study using AC maps from the PET/CT, Dixon, model A PET/MR, and model B PET/MR methods. Temporal and parietal hypometabolism consistent with underlying Alzheimer disease can be appreciated on surface maps derived from all methods, and the images would be sufficient for clinical diagnosis. Figure 2 and Table 1 offer a global summary of the magnitude and distribution

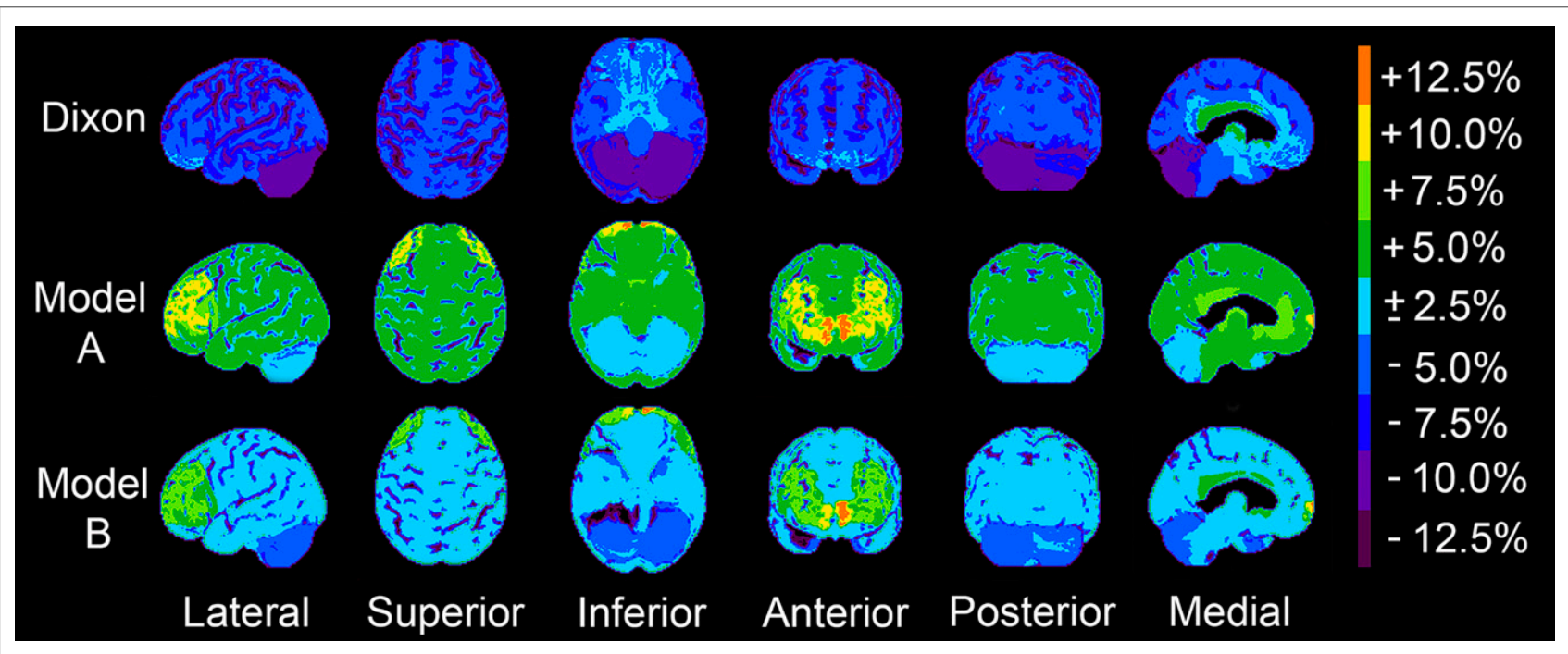

FIGURE 3. Surface maps of mean ${ }^{18} \mathrm{~F}-\mathrm{FDG}$ PET SUV bias between CT and MR-based AC methods $(n=16$ subjects, scale bar $=$ mean bias as percentage of CT SUV). First row demonstrates that Dixon-based AC underestimated SUV in most cortical regions but with little bias in basal and mesial temporal and frontal lobes. Atlas-based approach (model A) reduced overall bias but overestimated SUV in many cortical regions. Adjusting softtissue LAC for brain to $0.098 \mathrm{~cm}^{-1}$ (model B) reduced bias such that only cerebellar and rostral frontal lobes demonstrated potentially clinically significant bias (defined here as $>5 \%$ SUV estimation error). 


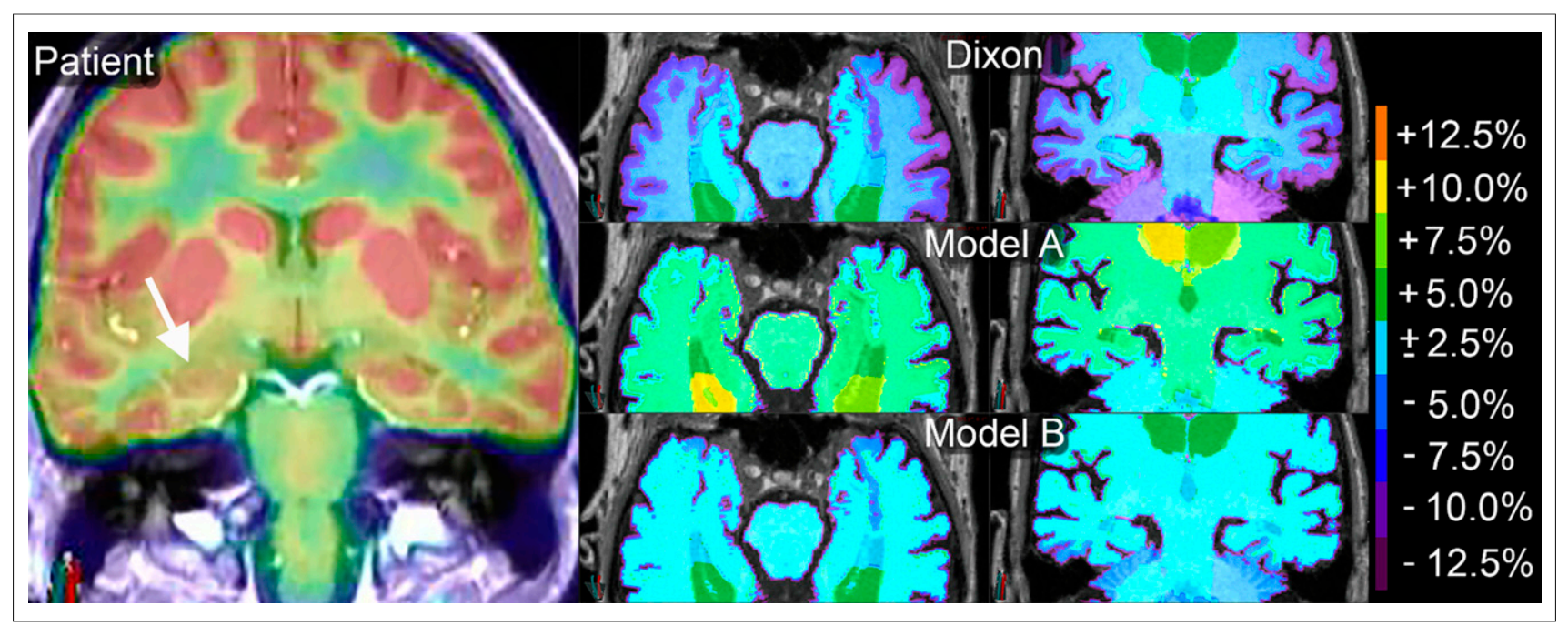

FIGURE 4. Estimation of mean bias for MR-based $A C$ methods in medial temporal lobe structures (color scale bar $=S \mathrm{SV}_{\text {mean }}$ bias compared with CT). First panel demonstrates cropped oblique coronal blended image of ${ }^{18} \mathrm{~F}-\mathrm{FDG}$ PET and MPRAGE for patient with MR-negative right medial temporal lobe epilepsy. There is subtle $8.9 \%$ asymmetric decrease in right hippocampal SUV compared with contralateral side (arrow). Corresponding coronal images of mean bias maps for all 16 subjects are shown for Dixon, model A, and model B MR AC methods. Dixon and model B AC maps demonstrate no clinically significant bias in the medial temporal lobe. Only slight asymmetry in SUV estimation error is seen for all 3 methods.

of SUV estimation biases for the 3 MR AC methods compared with reference $\mathrm{CT}$ AC obtained on the same day. There was a wide range of SUV estimation biases for the Dixon-based MR AC method and whole-brain $\mathrm{SUV}_{\text {mean }}$ underestimation. Incorporating a model of the bone compartment into the Dixon-based method reduces the magnitude and spread of regional mean estimation biases (model A). Altering the LAC in model $\mathrm{B}$ to reflect the attenuation of brain tissue improves accuracy; that is, whole-brain SUV estimation bias was reduced by $95 \%$ compared with Dixon alone, and only 5 remaining FreeSurfer regions still had SUV estimation bias of 5\% or greater ( $87 \%$ reduction, from 38 to 5). For simplicity, the following analysis and discussion emphasize SUV estimation biases that are $5 \%$ or greater in magnitude and statistically significant $(P<0.05)$ compared with reference CT AC. Up to 5\% differences might be expected for patients on different days or different PET/CT scanners (23).

Surface-based displays of the $\mathrm{SUV}_{\text {mean }}$ bias in Figure 3 demonstrate a global and relatively symmetric 5\%-10\% underestimation of cortical SUV throughout both cerebral hemispheres and the cerebellum for Dixon. Dixon-based SUV estimation in the medial and basal portions of the frontal and temporal lobes was accurate. Adding the anatomic model to the Dixon AC method (model A) conversely led to SUV overestimation throughout the cortex, but of lower magnitude. The largest-magnitude SUV estimation bias was within the frontal regions. A $2 \%$ reduced LAC for model B AC reduced the bias across the cortex and frontal regions further, but there remained some frontal-lobespecific overestimation biases.

Unlike neurodegeneration studies, interpretations of ${ }^{18} \mathrm{~F}-\mathrm{FDG}$ brain studies for epilepsy are more likely to depend on the recognition of subtle visual or quantitative SUV asymmetries, often located in deep temporal lobe structures not characterized by surface projections. Figure 4 shows cross-sectional axial and coronal maps of SUV estimation bias for the 3 MR-based AC methods through the deep and superficial structures of the medial temporal lobe, where most adult epilepsy abnormalities are found (24). The Dixon and model B approaches show little bias in the hippocampus, amygdala, entorhinal cortex, and parahippocampal gyri, whereas model A overestimates SUV in these regions. All 3 MR attenuation methods provide relatively symmetric data (Table 1 ).

\section{Individual Subject Factors That Correlate with MR AC Error}

Several individual anatomic features correlated with SUV estimations based on MR AC methods. The CT Hounsfield units in the basal ganglia negatively correlated with whole-brain mean bias for all 3 MR-based methods (e.g., for the Dixon method; $\mathrm{R}=$ $-0.69, P=0.003)$. Table 2 shows the impact of frontal and sphenoid sinus pneumatization on the 3 FreeSurfer regions for which model B SUV estimation had biases compared with CT. As frontal sinus pneumatization increased among the 16 subjects, model B SUV estimation error for the rostral middle frontal cortex

TABLE 2

Correlation Between Sinus Pneumatization and SUV mean Bias for Model B PET/MR AC Compared with Reference CT

\begin{tabular}{lccc}
\hline \multicolumn{1}{c}{ FreeSurfer region } & SUV $_{\text {mean }}$ bias & Sphenoid sinus pneumatization & Frontal sinus pneumatization \\
\hline Frontal pole & $+10.5 \%$ & $\mathrm{R}=+0.56(P=0.024)$ & $\mathrm{R}=-0.36(P=0.175)$ \\
Rostral middle frontal & $+6.1 \%$ & $\mathrm{R}=+0.46(P=0.073)$ & $\mathrm{R}=-0.55(P=0.027)$ \\
Pars triangularis & $+7.0 \%$ & $\mathrm{R}=+0.51(P=0.044)$ & $\mathrm{R}=-0.39(P=0.137)$
\end{tabular}

$n=16$ subjects; only left-sided data are shown for simplicity. 


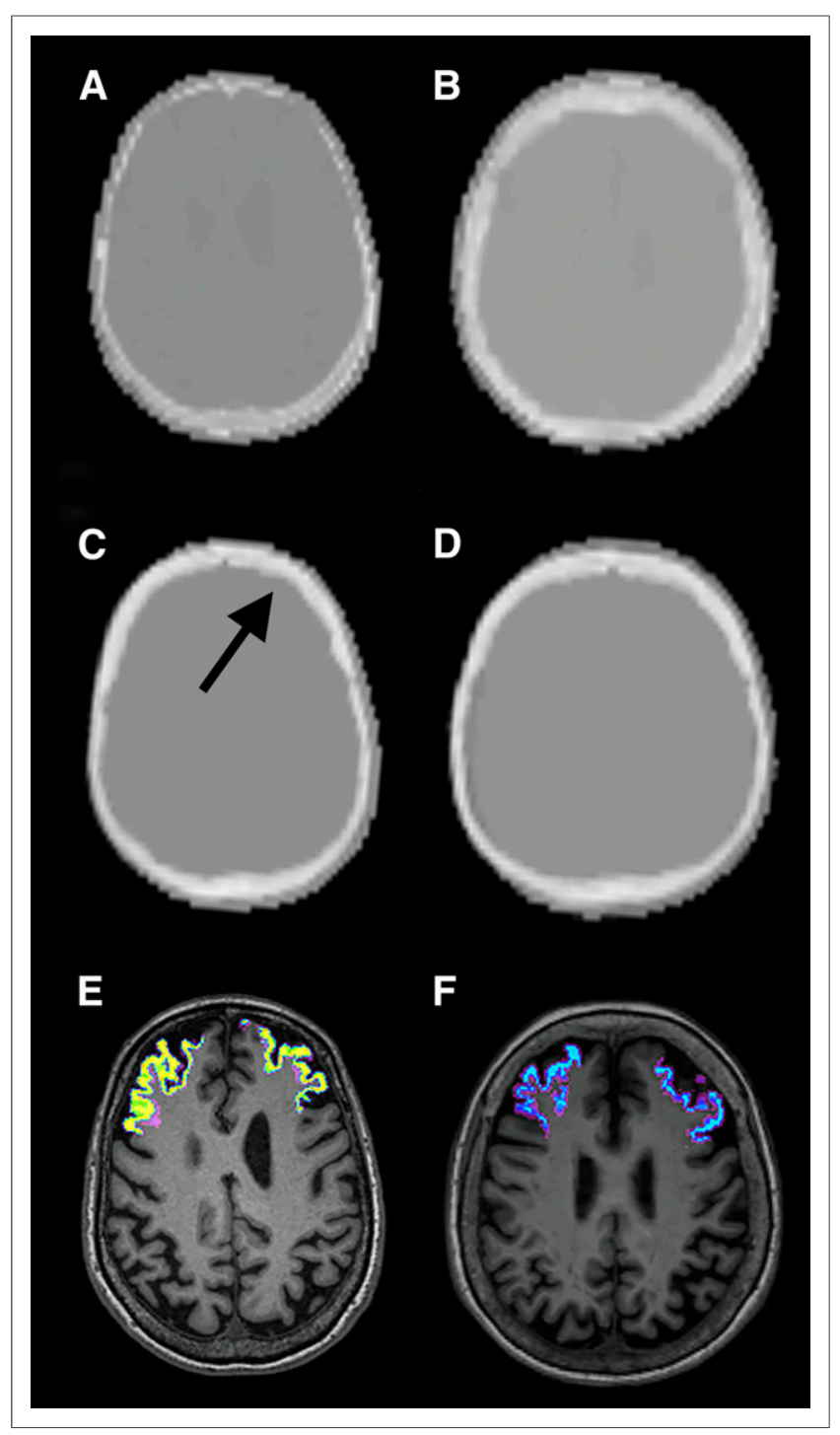

FIGURE 5. Visual comparison between $\mathrm{CT} \mu$-maps ( $\mathrm{A}$ and $\mathrm{B}$ ) and model $B \mu$-maps (C and D) for 2 individual subjects selected with high (left) and low (right) SUV bias in rostral middle frontal FreeSurfer region. First subject (column 1) had $8.0 \%$ mean bias (yellow region superimposed on axial MPRAGE, E) whereas second subject (column 2) had 3.6\% mean bias in these same bilateral frontal regions (blue region in F). Comparison of $\mu$-maps for first subject demonstrated that MR model B overestimated frontal calvarium thickness (arrow) whereas model $B \mu$-map (D) estimated frontal calvarium thickness more accurately for second subject.

actually decreased. Conversely, when sphenoid sinus pneumatization increased, model $\mathrm{B}$ overestimations in the 3 regions increased. The frontal pole also correlated with CT Hounsfield units for the clivus ( $R=-0.57, P=0.022$ ), a potential surrogate marker for overall skull base mineralization. Otherwise, no significant correlations were detected between the 3 regions of SUV estimation error for model B and the various other factors described in the Materials and Methods $(P>0.05)$.

Many other FreeSurfer regions displayed correlations between Dixon SUV biases and underlying individual anatomic features that are beyond the scope of this study. Additional subject factors that were characterized (age, dental amalgam, Alzheimer dementia diagnosis, PET/CT or PET/MR scanning time) did not significantly correlate with the mean bias for any of the MR AC methods.

\section{DISCUSSION}

This study demonstrated the benefits of modifying Dixon-based $\mu$-maps with a model-based bone compartment superimposed using common anatomic landmarks. The model-based approach reduced the whole-brain SUV estimation bias present in Dixon-only MR AC methods by $95 \%$, with the residual $\mathrm{SUV}_{\text {mean }}$ bias being similar to the reference $\mathrm{CT}$ AC of $-0.3 \%$ (Table 1). This result remained valid for nearly all individual FreeSurfer-parcellated brain regions, with only 5 of 91 FreeSurfer regions demonstrating a statistically significant SUV estimation bias of $5 \%$ or greater (an $87 \%$ reduction compared with the Dixon-only method) (Fig. 2; Table 1). There were few significant SUV estimation bias asymmetries using the model-based MR AC (Table 2), a useful feature for clinical interpretation of ${ }^{18} \mathrm{~F}$ FDG brain studies. The bone compartment model-based approach relies on a short Dixon sequence (19-s acquisition) without requiring additional MR sequences. The model B AC maps can be generated in 2-3 min and applied retrospectively to preexisting data. Although this study evaluated elderly subjects, the advantages of anatomy-based MR AC methods should be applicable to other patient populations common to PET studies, such as epilepsy (Fig. 4).

Previous reports tried to improve MR-based AC for integrated PET/MR studies with different atlas-based approaches. A combination of local pattern recognition and atlas registration to 3 subjects resulted in a residual $\mathrm{SUV}_{\text {mean }}$ bias of $3.2 \% \pm 2.5 \%$ in 12 ROIs compared with reference CT AC (11). If PET/MR AC is based on warping individual subject MR data to a populationbased atlas of coregistered CT and MR data to generate a pseudo-CT scan (13), the voxel-based absolute SUV estimation bias is $2.9 \% \pm 0.9 \%$ for simulated cases and approximately $5 \%$ for a real patient case compared with CT. The model B approach described here generated a slightly lower bias of $4.0 \% \pm 1.5 \%$ in 16 individual subjects when similar bias calculation methods were used (i.e., computed for the whole brain as segmented by FreeSurfer). IzquierdoGarcia et al. used statistical parametric mapping to coregister subject PET/MR data to an anatomic template (25). Voxel-based absolute error compared with $\mathrm{CT}$ with this method $(3.9 \% \pm 5.0 \%)$ is equivalent to absolute whole-brain bias error with model B.

The accuracy of any anatomic-model-based MR AC regional SUV estimation may be affected by common, individual-specific variations in innate skull or brain anatomy or by postsurgical changes to the skull base and calvarium. To characterize the impact of anatomic variation on model-based bone compartment modification of the Dixon MR AC method, we characterized the impact of brain, skull base, and calvarial features that are known to vary among individuals without a history of prior surgery. The largest region-specific SUV estimation biases with the model B method were in the frontal poles and rostral middle frontal gyri, similar to a previous atlas-based approach (25). We hypothesized that this reflected individual variation in frontal sinus pneumatization, but a negative correlation was present only for the rostral middle frontal region (such that increasing pneumatization decreased model B SUV overestimation bias). Conversely, model B SUV estimation bias both for this region and for the frontal poles positively correlated with sphenoid sinus pneumatization (Table 2). In a post hoc analysis, we then ranked the amount of SUV estimation bias between model B and reference CT for the frontal poles in all 16 subjects. Visual analysis of the $\mu$-maps demonstrated discordance between the superimposed bone compartment model and the CTmeasured thickness of the frontal calvarium in those subjects with the largest frontal pole SUV estimation error for model B (Fig. 5). 
Frontal bone thickness and pneumatization are highly variable in individual subjects, potentially limiting the pure atlas- or modelbased approaches to PET/MR AC.

Several additional subject-specific features correlated with SUV estimations for all $3 \mathrm{MR}$ AC methods, although not all were associated with an SUV estimation bias compared with CT. As measured CT Hounsfield units in the basal ganglia increase, whole-brain SUV underestimation for all 3 MR AC methods increases $(R=-0.69$ or lower, $P \leq 0.003)$. This requires independent verification in a larger dataset but suggests that the optimal LAC for brain parenchyma may depend on the health of the underlying tissue. This result and others suggest that clinical investigation for subtle SUV differences should account for limitations of the anatomic model for specific regions that vary among individual subjects. Further, future MR AC methods may need to derive data directly from individual patients (such as ultrashort echo time) and cannot rely solely on atlas-based approaches to further improve SUV estimation accuracy.

\section{CONCLUSION}

A Dixon-based MR AC with the addition of a model-based bone compartment and a $2 \%$ reduction in soft-tissue LAC improved whole-brain SUV estimation accuracy by $95 \%$. This approach gave a similar or better improvement in the accuracy of SUV estimation compared with other approaches $(13,25)$ but represents a prototype that does not require additional MR sequences. Besides being useful in patients with cognitive impairment, this new MR AC method should increase diagnostic accuracy for other clinical groups studied with ${ }^{18}$ F-FDG PET (e.g., epilepsy). Residual SUV overestimation biases in the polar and lateral frontal lobe regions appear to reflect individual-subject discordance between the bone compartment model and frontal calvarium thickness (not bone density or pneumatization), suggesting that a model-based MR AC approach may always produce some regional biases unless modified by sameday, direct MR data that characterize individual variation in skull anatomy well.

\section{DISCLOSURE}

The costs of publication of this article were defrayed in part by the payment of page charges. Therefore, and solely to indicate this fact, this article is hereby marked "advertisement" in accordance with 18 USC section 1734. This research was supported by the Center for Advanced Imaging Innovation and Research, a National Institute for Biomedical Imaging and Bioengineering Biomedical Technology Resource Center (NIH P41 EB017183). Timothy Shepherd received research support from the National Institute of Aging (NIH 1K23 AG048622-01). No other potential conflict of interest relevant to this article was reported.

\section{ACKNOWLEDGMENTS}

We thank Chris Glielmi, Kimberly Jackson, Bangbin Chen, and Hina Jaggi for their help.

\section{REFERENCES}

1. Catana C, Drzezga A, Heiss WD, Rosen BR. PET/MRI for neurologic applications. J Nucl Med. 2012;53:1916-1925.

2. Beyer T, Townsend DW, Brun T, et al. A combined PET/CT scanner for clinical oncology. J Nucl Med. 2000;41:1369-1379.

3. Keereman V, Mollet P, Berker Y, Schulz V, Vandenberghe S. Challenges and current methods for attenuation correction in PET/MR. MAGMA. 2013;26:81-98.

4. Martinez-Möller A, Souvatzoglou M, Delso G, et al. Tissue classification as a potential approach for attenuation correction in whole-body PET/MRI: evaluation with PET/CT data. J Nucl Med. 2009;50:520-526.

5. Drzezga A, Souvatzoglou M, Eiber M, et al. First clinical experience with integrated whole-body PET/MR: comparison to PET/CT in patients with oncologic diagnoses. J Nucl Med. 2012;53:845-855.

6. Defrise M, Rezaei A, Nuyts J. Time-of-flight PET data determine the attenuation sinogram up to a constant. Phys Med Biol. 2012;57:885-899.

7. Catana C, van der Kouwe A, Benner T, et al. Toward implementing an MRIbased PET attenuation-correction method for neurologic studies on the MR-PET brain prototype. J Nucl Med. 2010;51:1431-1438.

8. Aitken AP, Giese D, Tsoumpas C, et al. Improved UTE-based attenuation correction for cranial PET-MR using dynamic magnetic field monitoring. Med Phys. 2014;41:01232.

9. Keereman V, Fierens Y, Broux T, Deene YD, Lonneux M, Vandenberghe S. MRI-based attenuation correction for PET/MRI using ultrashort echo time sequences. J Nucl Med. 2010;51:812-818.

10. Delso G, Carl M, Wiesinger F, et al. Anatomic evaluation of 3-dimensional ultrashort-echo-time bone maps for PET/MR attenuation correction. $J$ Nucl Med. 2014;55:780-785.

11. Hofmann M, Steinke F, Scheel V, et al. MRI-based attenuation correction for PET/MRI: a novel approach combining pattern recognition and atlas registration. J Nucl Med. 2008;49:1875-1883.

12. Johansson A, Garpebring A, Asklund T, Nyholm T. CT substitutes derived from MR images reconstructed with parallel imaging. Med Phys. 2014;41:08232.

13. Burgos N, Cardoso MJ, Thielemans K, et al. Attenuation correction synthesis for hybrid PET-MR scanners: application to brain studies. IEEE Trans Med Imaging. 2014;33:2332-2341.

14. Paulus DH, Quick HH, Geppert C, et al. Whole-body PET/MR imaging: quantitative evaluation of a novel model-based MR attenuation correction method including bone. J Nucl Med. 2015;56:1061-1066.

15. Wahlund LO, Barkhof F, Fazekas F, et al. A new rating scale for age-related white matter changes applicable to MRI and CT. Stroke. 2001;32:13181322 .

16. Kinahan PE, Townsend DW, Beyer TT, Sashin D. Attenuation correction for a combined 3D PET/CT scanner. Med Phys. 1998;25:2046-2053.

17. Zhan Y, Zhou XS, Peng Z, Krishnan A. Active scheduling of organ detection and segmentation in whole-body medical images. Med Image Comput Assist Interv. 2008;11(Pt 1):313-321.

18. Hermosillo G, Chefd'Hotel C, Faugeras O. Variational methods for multimodal image matching. Int J Comput Vis. 2002;50:329-343.

19. Dale AM, Fischl B, Sereno MI. Cortical surface-based analysis. I. Segmentation and surface reconstruction. Neuroimage. 1999;9:179-194.

20. Fischl B, Salat DH, Busa E, et al. Whole brain segmentation: automated labeling of neuroanatomical structures in the human brain. Neuron. 2002;33:341-355.

21. Jenkinson M, Bannister P, Brady M, Smith S. Improved optimization for the robust and accurate linear registration and motion correction of brain images. Neuroimage. 2002;17:825-841.

22. Jenkinson M, Smith S. A global optimisation method for robust affine registration of brain images. Med Image Anal. 2001;5:143-156.

23. Adams MC, Turkington TG, Wilson JM, Wong TZ. A systematic review of the factors affecting accuracy of SUV measurements. AJR. 2010;195:310-320.

24. Téllez-Zenteno JF, Hernández-Ronquillo L. A review of the epidemiology of temporal lobe epilepsy. Epilepsy Res Treat. 2012;2012:630853.

25. Izquierdo-Garcia D, Hansen AE, Förster S, et al. An SPM8-based approach for attenuation correction combining segmentation and nonrigid template formation: application to simultaneous PET/MR brain imaging. J Nucl Med. 2014;55:18251830 